\title{
Research of Network System based on Routing Exchange Platform Peng Xiao ${ }^{1, a}$ \\ ${ }^{1}$ Jiangxi Vocational College of Industry\& Engineering, Pingxiang, Jiangxi, 337000 \\ ${ }^{a}$ email
}

\author{
Keywords: Network System, Routing Exchange Platform, Reconstruction Network
}

\begin{abstract}
Traditional single information network scalability and relative stability can not adapt to the dynamic development of the next generation network services. To solve this problem, the concept of reconfigurable network is put forward and the network can be constructed to support a plurality of different network system logic bearer network on the same physical network resources. In the process of rapid development and updates, the configurable network can maintain a low probability of network transformation, the network business support robustness and scalability will be improved significantly.
\end{abstract}

\section{Introduction}

Traditional dynamic information network development, it is difficult to lead a comprehensive and effective network behavior analysis, network traffic so that it carries the diversity and uncertainty of the feature that the next generation is not sufficient research network and network user to the next there is a contradiction between the growing generation network needs. The contradiction consists of two aspects: one contradiction diversity single scalable network architecture and existing network traffic between; on the other hand, the relative stability of the network architectures and future network traffic needs not contradiction between certainty and uncertainty.

To alleviate this contradiction, in general, there are two solutions. First, relying on expanding the link bandwidth, improve processing speed and capacity of the node and other methods, providing sufficient network resources for the new network services; the second is to ensure that the main business of the network at the same time, by increasing the complex control algorithms and protocols and other technologies to repair the way to solve the service quality of new business. These measures belong to a network of business-oriented development model, only for a particular type of business to build the network, not only difficult to meet the growing differences in characteristics of customers' business needs, but also paid a price increase network complexity and rapid information transmission efficiency significantly reduced.

\section{Framework Design of Routing Switching Platform}

Traditional telecommunication network, broadcast and Internet are used to construct a logical bearer network special network mode on a physical network, to provide services for special network services, such as telecommunications networks and services for voice telephony services, wide network serving the radio and television service, Internet services and other data transmission services. Private network dedicated business networking mode in favor of its bearer network services provide guaranteed quality of service, but a waste of physical network resources. In order to improve the utilization of network resources, network operators have converged in a variety of business logic bearer network. However, the logic bearer network together with tight relationship Woe main business network can not ensure that the original network complexity, while providing quality assurance services for the newly added network services. That is to build a logical bearer network on a physical network and provide service for a variety of networking services on the network can not meet the network traffic variability dynamic development needs.

To support multiple network building reconfigurable logic bearer network on the same physical network resources, FPGA-based RTPR, and establishing a general framework for reconfigurable routing and switching platform, the framework includes reconstruction management plane, control 
plane, switching plane, forwarding plane connecting link, access and flat planes of each. Reconfigurable routing and switching platform is from the reconstructed frame dimensions, size and deformation mechanisms of reconstruction three-pronged approach to ensure that the platform is flexible enough and low complexity. Among them, the reconstruction of the region is to determine the dimensions of the platform, the platform to determine particle size reconstruction of the constituent elements of the reconstruction levels.

Reconfigurable network comprises a plurality of logical bearer network. Similarly, reconfigurable routing and switching platform also includes a plurality of logical node carrying. Each node provides a different logical bearer network service and network processing nodes is constructed to provide support to the logic bearer network. To establish the overall framework for building multiple logical nodes carrying reconfigurable routing and switching platform provides a reconfigurable environment. Under Reconstruction size dimensions and conditions determined in the form of a detailed description of examples of the reconfigurable routing switch platform deformation mechanisms, including initialization and reconstruction of both platforms. The deformation mechanism under the coordination of the reconstruction of the management plane, divided into three steps, first exchange between the plane and each plane connected to the link to create one or more switching components as the center plane between the connecting link network traffic transmission path reconstruction framework; the second is the hardware and software components loaded on the control and forwarding plane, each reconstructed frame; the third is the reconstructed configuration, insert the corresponding plug and play components on the access plane and ultimately generate a plurality of logical carrier routing nodes reconfigurable switching platform.

\section{Network System Reconstruction}

For a service-oriented network technology system provides a layered architecture, top-down structure is three layers: application layer traffic, service access / control layer, the network layer service. Network Service layer consists of three sub-layers: a logical bearer layer, reconfigurable edge layer and resource sharing layer.

With this layered system and establish a loose coupling between the user and the network service business relationships in a hierarchical manner to provide network services to support a given category of user traffic generation network services. Produce a variety of characteristics in the business service application layer, user applications, mobile terminals, fixed telecommunication terminal, a computer will be. In the logic bearer layer, by matching the characteristics of the business, quality of service parameters to service access / control layer provides QoS guarantee, and based on business clusters is proposed to construct a certain service features to meet the needs of the logical network; in reconfigurable edge layer, according to the logic bearer layer needs to build new properties to meet the business, the ability to have the required service logic bearer network, or no longer need to release a logical bearer network. Resource sharing network layer provides the physical transmission and processing resources nodes, the node processing resources resource sharing layer is based on open reconfigurable routing and switching platform technology to build routing and switching node system platform. In such a system to ensure technology by business characteristics, quality of service parameters matching and optimization of network resources to form a network with a certain service providers the ability to meet quality of service requirements of the business. Open-based reconfigurable routing and switching technology platform for new network system, can more effectively provide a flexible network services, provide quality assurance for the business needs of different categories.

Thus, the network is no longer carried out according to specific business to build, but on the ability to provide network services, network services provide the ability for the performance of the network can provide network services types, each of which can support a variety of network services similar characteristics to business users and emerging business also has a smooth interface appears most emerging businesses can take advantage of existing network support, only a few emerging business needs new network provides network services. Therefore, it can reduce the 
network traffic generated by the new passive transformation probability.

New network technology system to solve the core issue-oriented service delivery technical ideas are: to build a resource can be shared, reconfigurable nodes, capable of providing a flexible service physical network, create demand for new network services through a network reconstruction node implementation, in order to enhance service delivery network scalability.

All kinds of traffic from the service access, control layer into the network service layer, network layer service traffic service providers are stratified, mainly consists of the following steps.

After receiving the logic bearer layer in bearer network from the service access logic, control layer request for transmission resource and the shared resource node processing layer resource capabilities of the queries are parameters selected by the logic bearer layer according to business needs characteristics, information including the logical bearer network processing node input/output interface information, the bandwidth distribution logic bearer network diameter maximum number of hops, the logical bearer network type and protocol type supported by the input/output interface and the like.

Resource sharing reconfigurable routing layer switching platform based on the query request, the logic bearer layer reporting capabilities.

According to the business logic layer bearing demand characteristics, quality of service parameters and network resources to determine whether they have to build conditional logic bearer network, and if so, then select the appropriate category of business networking strategies to the reconfigurable logic edge layer issued bearer network Construction command.

After the logic bearer network was successfully constructed, according to the logic bearer layer access / control layer of demand, changes in resource sharing resources and other layers of logic bearer network management and maintenance, to ensure service delivery.

The access logic bearer layer / layer control requirements, determine the duration of the logical bearer network, if there is no demand for the service creation services, on the edge layer reconfigurable issued revocation orders.

Reconstruction edge layer according to the command logic bearer layer, releasing the logical bearer network resource, the revocation of the logic bearer network no longer generate traffic for the service provided. By the above-described network system design services for the offer, based on a unified, open standard components and components, design of network node equipment, and processing tasks for different components or components reconfigurable design quickly to meet new business needs for network equipment, which will help telecommunications network, network, Internet three network convergence, is conducive to a variety of flexible business conduct conducive to high-end routing and switching equipment standardized, open technology.

\section{Conclusion}

Traditional dynamic information network development is difficult to lead a comprehensive and effective network behavior analysis, it carries the diversity and uncertainty of the feature that the next generation is not sufficient and network traffic network study of dynamic there is a contradiction between the developments. The contradiction consists of two aspects: one contradiction diversity single scalable network architecture and existing network traffic between; on the other hand, the relative stability of the network architectures and future network traffic needs not contradiction between certainty and uncertainty. To solve the above problems, a concept of reconfigurable network is put forward. Reconfigurable network user business cluster and network service isolation, can build a variety of resources on a physical network resources can be shared, node reconfigurable logic bearer network provides a flexible network services. A logical bearer network to generate a network service provided to a class of similar characteristics of network services using the network. The method can maintain a low probability of network transformation for the new network services, build and deploy services quickly and provide an experimental platform to achieve. Reconfigurable network includes network routing transmission links and reconfigurable switching platform. However, current research is still confined to the high-performance router forwarding or control of a single reconfigurable technology, lack of 
internet exchange and the overall framework for reconfigurable routing switching technology theoretical research and engineering, has seriously hindered the reconfigurable network deployment. We think future research is meaningful for the network development.

\section{Acknowledgements}

Jiangxi Province, Department of Education 2015 scientific and technological research projects (GJJ151274), 2015 Pingxiang Science and Technology Support Program (Central Region cloud-based animation rendering service platform research and development), education examination and enrollment system reform in Jiangxi Province scientific research projects (No. : 1524)

\section{References}

[1] Network Performance Objectives for IP Based Services. ITU-T Recommendation Y 1541. 2002

[2] J.S. Turner, D.E. Taylor. Diversify the Internet. IEEE Globecom. 2005

[3] Gottlieb. A Comparative Study of Extensible Routers. Proc of the 2002 IEEE Open Architectures and Network Programming Conf. 2002

[4] Handley. Xorp: An Open Platform For Network Research ACM SIGCOMM Computer Communication Review 2003.

[5] Andrew W Moore, Konstantin A Papa Gianna K. Toward the Accurate Identification of Network Applications. Lecture Notes in Computer Science. 2005. 